\title{
The Fifth Edition of Diagnostic and Statistical Manual of Mental Disorders (DSM-5): What is New for the Pediatrician?
}

\author{
*Neetu Sharma, "Ruchi Mishra and Devendra Mishra \\ From Departments of Pediatrics, *GR Medical College, Gwalior, MP; \#ESIPGIMER, Basaidarapur, New Delhi; and Maulana Azad \\ Medical College, Delhi; India. \\ Correspondence to: Dr Devendra Mishra, Professor, Department of Pediatrics, Maulana Azad Medical College and Lok Nayak Hospital, Delhi \\ 110 002, India.drdmishra@gmail.com
}

T The Diagnostic and Statistical Manual of Mental Disorders (DSM) is the most important resource for the purpose of psychiatric diagnoses, whether by the psychiatrist or the general practitioner. The American Psychiatric Association published the fifth edition of DSM (DSM-5) [1], containing significant changes in the diagnostic criteria for certain disorders from those in DSM-IV, which had been in widespread clinical use for nearly two decades [2]. DSM-5 was developed over a six-year official process utilizing the contributions of more than 400 multi-disciplinary professionals from varied specialties from across the world.

Pervasive developmental disorders (Autism spectrum disorder), Attention-deficit/hyperactivity disorder and Global developmental delay/Mental retardation are common childhood neurodevelopmental disorders and comprise the bulk of referrals to Child Development Centers [3]. We herein enlist the major diagnostic changes in DSM-5 (Table I).

\section{Autism}

Autism is now being increasingly recognized to be a common neurodevelopmental disorder, with prevalence approaching $1 \%$ of the population [1]. In DSM-IV, deficits in three core domains were required for the diagnosis of Autism viz., Reciprocal social interactions, Restricted and repetitive behaviors or interests, and Verbal and nonverbal communication. As per DSM-5, deficits in only the first two core domains are required for diagnosis viz., Deficits in social communication and social interaction (as manifested by deficit in socialemotional reciprocity, deficit in nonverbal communicative behavior used for social interaction, and deficit in developing, maintaining, and understanding relationships); and, Restrictive, repetitive pattern of behavior, interest, or activities, (as manifested by at least two of: stereotyped or repetitive motor movements, use of objects, speech; insistence on sameness, inflexible adherence to routines, or ritualized patters of verbal or nonverbal behavior; highly restricted, fixated interests that are abnormal in intensity or focus; hyper- or hyporeactivity to sensory input or unusual interest in sensory aspects of the environment) [1]. Thus delay in language development is no longer required for diagnosis.

Another important change has been that Autism spectrum disorder (ASD) has been introduced as a new term in DSM-5 and covers all the previous four DSM-IV diagnoses viz., Autistic disorder (autism), Asperger's disorder, Childhood disintegrative disorder, and Pervasive developmental disorder-NOS [2].

Sensory behaviors, which were not a part of the diagnostic criteria in DSM-IV, have now been added. The previous classification did not specify symptom severity, which has now been defined for each area of the diagnostic criteria. A new entity, Social Communication Disorder has been introduced for children who fulfill all three social criteria of ASD but do not have any features of the repetitive and restrictive behavior criteria [1]. Rett's disorder is now considered as an associated known genetic condition. The requirement for appearance of symptoms prior to the age of three years, as given in DSM-IV [2] has now been replaced with "symptoms begin in early childhood", with the clause that "symptoms may not be fully manifest until social demands exceed capacity"[1]. It has also been emphasized that those already diagnosed as per DSM-IV, do not require any reevaluation.

\section{Attention-Deficit/Hyperactivity Disorder}

Attention-deficit Hyperactivity Disorder (ADHD) is also a common disorder, with population surveys suggesting that it occurs in about $5 \%$ of children and $2.5 \%$ adults across the globe [1]. In DSM-5, the text description of all the symptoms has been retained, but it has been suggested 
TABLE I CHANGE IN DSM-5 FROM DSM-IV-TR

DSM-IV DSM-5

Autism

- Category

Four subcategories: Autistic disorder; Asperger syndrome; Pervasive developmental disorder - Not otherwise specified; and Disintegrative disorder.

- Case Symptoms/Areas of impairement

Social reciprocity; Communicative intent; and

Restricted repetitive behaviors.

- Symptom severity: Not specified.

- Sensory behaviors: Not included in criteria.

-Appearance of symptoms: Requires that symptoms begin

prior to the age of 3 years; and symptoms must cause functional impairment.

Attention Deficit/Hyperactivity Disorder

-Age of diagnosis: arbitrarily set at 7 yrs

- Number of criteria for diagnosis: six for both hyperactivity and impulsivity

- Exemplification of criteria: criteria just mentioned, not exemplified.

- Pervasive disorders were previously an exclusion criterion.

- Subtyped into predominantly hyperactive, predominantly impulsive or both types co-existing.

that ADHD is not limited to childhood and may extend across the whole life span causing impairment even later in life. The age of diagnosis of ADHD, which was previously set at seven years [2] has been changed to the description "several inattentive or hyperactive-impulsive symptoms were present prior to age 12" [1]. This has been done because it was found that many children had onset of symptoms even after the age of seven years. Moreover, a good recall by adult of the childhood symptoms at less than seven years is often unreliable. It was recently shown that if the age of recall of symptoms was increased from 7 years to 12 years, the percentage of people who could recollect their symptom onset increased from $50 \%$ to $95 \%$ [4]. Concurrently, the number of criteria for the diagnosis in adolescents and adults has been lowered to five symptoms both for inattention as well as for hyperactivity and impulsivity (as opposed to six in DSM-IV). This has been done as there is research evidence for clinically significant impairment due to ADHD with the cut-off at five symptoms [4]. Pervasive disorders were previously an exclusion criterion, but a co-morbid diagnosis with ASD is now allowed.
All are combined into one term - Autism Spectrum Disorders

Deficits in social communication and social interaction; and

Restricted, repetitive patterns of behavior, interests, or activities

Defined for each area of diagnostic criteria.

Added in the criteria

Symptoms begin in early childhood, with the clause that

"symptoms may not be fully manifest until social demands exceed capacity”

"several inattentive or hyperactive-impulsive symptoms were present prior to age 12 ”

Reduced to 5 for both hyperactivity and impulsivity

Examples of all the 18 symptoms have been described for all age ranges.

Co-morbid diagnosis with ASD is now allowed.

Subtyping has been replaced by variable presentation.

\section{Mental Retardation/InTELletual Disability}

Reflecting the increasing use of the term in professional and lay literature, Intellectual disability is the new term in DSM-5 replacing the term Mental retardation in DSM-IV. The previous version classified the severity on the basis of the cognitive capacity (Intelligence quotient, IQ), but DSM-5 specifies that severity is to be determined by adaptive functioning rather than by IQ score [1]. Global developmental delay is diagnosed in "individuals who are unable to undergo systematic assessment of intellectual functioning” including children younger than five years, when clinical severity cannot be reliably assessed during early childhood [1].

\section{DSM-5: Advantages And Pitfalls}

International Classification of Diseases (ICD-11) is likely to be introduced next year, and DSM-5 has been developed to have a greater harmony with ICD-11, though that needs to be confirmed after widespread clinical and research use. DSM-5 has also given more emphasis to issues important to diagnosis and clinical care like gender and culture on the presentation of the disorder [1]. The 
multi-axial diagnosis, a characteristic feature of earlier editions has also been given the go by, as this was incompatible with the diagnostic system in rest of medicine.

There has been some concern in recent publications on the implications of these changes, especially related to the more stringent ASD diagnostic criteria [5], but some positive aspects have also been identified [6]. Field studies have shown that for autism and ADHD, clinicians in general agree with which patients meet DSM-5 diagnostic criteria; though, for some other conditions there is less agreement [7]. Those interested in more details of DSM-5 can refer to the full document [1], or to an online resource with an extended description of all changes (www.psychiatry.org/dsm5) [1]. However, the major hindrance to the immediate application of these criteria in clinical practice will probably be the availability of diagnostic tools in agreement with the new criteria. The recently developed Indian tools for ASD [8] and ADHD [9] will also need to measure up.

Contributors: All authors were equally involved in all aspects of the manuscript preparation.

Funding: None; Competing Interests: None stated.

\section{REFERENCES}

1. American Psychiatric Association. Diagnostic and
Statistical Manual of Mental Disorders, 5th ed. Arlington, VA American Psychiatric Publishing; 2013.

2. American Psychiatric Association. Diagnostic and Statistical Manual of Mental Disorders (4th ed., Text Revision). Washington, DC: 2000.

3. Jain R, Juneja M, Mishra D. Referral profile of a child development clinic in Northern India. Indian J Pediatr. 2012;79:602-5.

4. Barkley RA, Brown TE. Unrecognized Attention-deficit/ hyperactivity disorder in adults presenting with other psychiatric disorders. CNS Spectr. 2008;13:977-84.

5. Frazier TW, Youngstrom EA, Speer L, Embacher R, Law $\mathrm{P}$, Constantino J, et al. Validation of proposed DSM-5 criteria for autism spectrum disorder. J Am Acad Child Adolesc Psychiatry. 2012; 51:28-40.

6. McPartland JC, Reichow B, Volkmar FR. Sensitivity and specificity of proposed DSM-5 diagnostic criteria for autism spectrum disorder. J Am Acad Child Adolesc Psychiatry. 2012;51:368-83.

7. Kupfer DJ, Kuhl EA, Regier DA. DSM-5-the future arrived. JAMA. 2013;309:1691-2.

8. Juneja M, Mishra D, Russell PSS, Gulati S, Deshmukh V, Tudu $\mathrm{P}$, et al. INCLEN diagnostic tool for Autism Spectrum Disorder (INDT-ASD): Development and validation. Indian Pediatr. 2014;51:359-65.

9. Mukherjee S, Aneja S, Russell P, Gulati S, Deshmukh V, Sagar R, et al. INCLEN diagnostic tool for Attention Deficit Hyperacticity Disorder (INDT-ADHD): Development and validation. Indian Pediatr. 2014;51: 457-62.

\section{ADDITIONAL REVIEWERS 2014}

INDIAN PEDIATRICS

Volume 51, November 19, 2014 - December 31, 2014

The present status of this Journal is largely dependent on the expertise and selfless cooperation of the following Reviewers whose help we gratefully acknowledge. We are indebted to them for this service. -Journal Committee

Solakshana Shridhar Baliga

Bidisha Banerjee

Ginnela Brahmam

Lavjay Butani

Vaishali Ranjit Deshmukh
Shuichi Ito

Prachi Jain

Jai Pal Kapoor
Sunita Ojha

Sunil Natha Mhaske

Asha Moudgil

Yuan Shi

Shakal Narayan Singh 